Published in final edited form as:

Cancer Chemother Pharmacol. 2008 March ; 61(3): 423-433. doi:10.1007/s00280-007-0485-9.

\title{
Phase I and pharmacokinetic study of UCN-01 in combination with irinotecan in patients with solid tumors
}

\author{
Antonio Jimeno, \\ The Sidney Kimmel Comprehensive Cancer Center, Johns Hopkins University, Cancer Research
} Building I, 1650 Orleans Street, Room 186, Baltimore, MD, USA

\section{Michelle A. Rudek,}

The Sidney Kimmel Comprehensive Cancer Center, Johns Hopkins University, Cancer Research Building I, 1650 Orleans Street, Room 186, Baltimore, MD, USA

\section{Thomas Purcell,}

The Sidney Kimmel Comprehensive Cancer Center, Johns Hopkins University, Cancer Research Building I, 1650 Orleans Street, Room 186, Baltimore, MD, USA

\section{Daniel A. Laheru,}

The Sidney Kimmel Comprehensive Cancer Center, Johns Hopkins University, Cancer Research Building I, 1650 Orleans Street, Room 186, Baltimore, MD, USA

\section{Wells A. Messersmith,}

The Sidney Kimmel Comprehensive Cancer Center, Johns Hopkins University, Cancer Research Building I, 1650 Orleans Street, Room 186, Baltimore, MD, USA

\author{
Janet Dancey, \\ Cancer Therapy Evaluation Program, National Cancer Institute, Bethesda, MD, USA
}

Michael A. Carducci,

The Sidney Kimmel Comprehensive Cancer Center, Johns Hopkins University, Cancer Research Building I, 1650 Orleans Street, Room 186, Baltimore, MD, USA

\section{Sharyn D. Baker,}

The Sidney Kimmel Comprehensive Cancer Center, Johns Hopkins University, Cancer Research Building I, 1650 Orleans Street, Room 186, Baltimore, MD, USA

\section{Manuel Hidalgo, and}

The Sidney Kimmel Comprehensive Cancer Center, Johns Hopkins University, Cancer Research Building I, 1650 Orleans Street, Room 186, Baltimore, MD, USA

\section{Ross C. Donehower}

The Sidney Kimmel Comprehensive Cancer Center, Johns Hopkins University, Cancer Research Building I, 1650 Orleans Street, Room 186, Baltimore, MD, USA

\author{
Abstract \\ Purpose-7-Hydroxystaurosporine (UCN-01) is a protein kinase inhibitor that inhibits several \\ serine-threonine kinases including PKC and PDK1. Due to the preclinical synergistic effects seen \\ with topoisomerase I inhibitors and non-overlapping toxicity, UCN-01 and irinotecan were

\footnotetext{
(C) Springer-Verlag 2007

Correspondence to: Ross C. Donehower.

donehro@jhmi.edu.

Presented in part at the 16th EORTC-NCI-AACR Symposium on Molecular Targets and Cancer Therapeutics, September 2004.
} 
combined in a dose-finding study designed to determine the maximum tolerated dose (MTD), toxicity profile, and pharmacokinetics (PK) of UCN-01 and irinotecan.

Methods-Patients with incurable solid malignancies received UCN-01 intravenously (IV) as a 3-h infusion on day 1 and irinotecan IV over $90 \mathrm{~min}$ on days 1 and 8 of a 21-day cycle. Doses of UCN-01 for subsequent cycles were half the starting dose. Dose level 1 (DL1) consisted of $\mathrm{UCN}-01$ and irinotecan doses of 50 and $60 \mathrm{mg} / \mathrm{m}^{2}$, respectively. Blood samples were collected in cycle 1 for UCN-01, irinotecan, and irinotecan metabolites.

Results-A total of 16 patients were enrolled on the trial at UCN-01/Irinotecan doses of 50/60 $\mathrm{mg} / \mathrm{m}^{2}$ (DL1; $n=1$ ), $70 / 60 \mathrm{mg} / \mathrm{m}^{2}$ (DL2; $n=6$ ), $90 / 60 \mathrm{mg} / \mathrm{m}^{2}$ (DL3; $n=4$ ), and $70 / 90 \mathrm{mg} / \mathrm{m}^{2}$ (DL4; $n=5$ ). Two dose-limiting toxicities were observed each in DL3 and DL4 (2 grade 3 hypophosphatemia, 1 grade 4 hyperglycemia and grade 3 hypophosphatemia, 1 grade 4 febrile neutropenia). Fatigue, diarrhea, nausea, and anorexia were the most prevalent toxicities. No objective responses were documented, and four patients had stable disease for at least ten cycles. The long half-life $(292.0 \pm 135.7 \mathrm{~h})$, low clearance $(0.045 \pm 0.038 \mathrm{1} / \mathrm{h})$, and volume of distribution $(14.3 \pm 5.91)$ observed for UCN-01 are consistent with prior UCN-01 data. There was a significant decrease in $C_{\max }$ of APC, AUC of APC and SN-38, and AUC ratio of SN-38:irinotecan when comparing days 1 and $8 \mathrm{PK}$.

Conclusions-APC and SN-38 exposure decreased when administered in combination with UCN-01. The MTD of the combination based on protocol criteria was defined as $70 \mathrm{mg} / \mathrm{m}^{2}$ of $\mathrm{UCN}-01$ on day 1 and $60 \mathrm{mg} / \mathrm{m}^{2}$ of irinotecan on days 1 and 8 in a 21-day cycle.

\section{Keywords}

UCN-01; Irinotecan; Phase I; Cell cycle; G2/M checkpoint

\section{Introduction}

7-Hydroxystaurosporine (UCN-01) (Fig. 1) has been shown to be a selective inhibitor of a number of serine-threonine kinases, including the $\mathrm{Ca}^{2+}$ - and phospholipid-dependent protein kinase C (PKC), cell cycle-specific kinases [29, 30], Chk1 [11, 36] and 3phosphoinositide-dependent protein kinase-1 (PDK1) [7, 19]. UCN-01 was selected for clinical development because of its potent antiproliferative activity in vitro in the NCI human tumor cell screen against several cell lines and its antineoplastic activity in xenograft systems. While UCN-01 is a potent inhibitor of several important kinases, the precise mechanism of action for its antitumor activity is still not fully understood. In numerous preclinical studies it was documented that UCN-01 causes arrest of cell cycle progression at $\mathrm{G}_{1} / \mathrm{S}$ phase and/or abrogation of arrest at $\mathrm{G}_{2}$ phase at concentrations that reduce $\mathrm{PKC}$ activity, although the extent to which PKC or PDK1 modulation contributes to these effects remains undetermined.

Induction of apoptosis by UCN-01 has been reported in various human cell lines, including MCF-7 and MDAMB453 breast carcinoma [27], A431 epidermoid carcinoma [1], A549 NCSLC [16], U-87 and SG-388 glioma [3], and SBC-3 small cell lung cancer lines [34]. $\mathrm{UCN}-01$ has also been shown to enhance the cytotoxic properties of a variety of agents in human tumor cell lines [23]. At clinically relevant concentrations (20-150 nM), UCN-01 was shown to act synergistically with thiotepa, mitomycin $\mathrm{C}$, cisplatin, melphalan, topotecan, gemcitabine, fludarabine, and 5-fluorouracil. In particular, preclinical studies with UCN-01 and topoisomerase inhibitors demonstrate a synergy that is not sequence dependent [33]. 
Phase I studies of UCN-01 evaluating schedules of 72-and 3-h intravenous (IV) infusions have been completed $[9,25]$. Pharmacokinetic data from these studies revealed that the agent binds tightly to human alpha-1 acid glycoprotein (AAG) resulting in a long half-life of several weeks, small volume of distribution at steady state and low systemic clearance. To prevent drug accumulation UCN-01 dose was reduced by half after the first cycle in both schedules. The maximum tolerated dose (MTD) of UCN-01 for the 72-h schedule was 42.5 $\mathrm{mg} / \mathrm{m}^{2} /$ day for 3 days during cycle 1 and the same dose per day for 1.5 days for subsequent cycles. For the 3-h schedule, the cycle 1 MTD is $95 \mathrm{mg} / \mathrm{m}^{2}$ of UCN-01. Significant toxicities seen with this agent include hyperglycemia with metabolic acidosis, nausea, vomiting, and hypotension. Pulmonary toxicity was seen on the 72-h infusion phase I, and although in the 3-h trial of single agent UCN-01 no pulmonary toxicity or dyspnea was docu mented, the current combination study required adequate respiratory function as entry criteria.

These single-agent studies suggest that at doses close to the MTD, free UCN-01 concentrations are in the range predicted to enhance the antitumor effect of topoisomerase inhibitors. A combination of UCN-01 and topotecan has been recently reported, which showed good tolerance and preliminary evidence of clinical activity [14]. Due to potential synergy and non-overlapping toxicity profiles, $\mathrm{UCN}-01$ and irinotecan were combined in a dose-finding phase I study, designed to determine the MTD, acute and chronic toxicity profile, and pharmacokinetics (PK) of UCN-01 and irinotecan.

\section{Patients and methods}

\section{Patient eligibility}

Patients were required to have histologically confirmed malignancy that was metastatic or unresectable and for which standard curative or palliative measures did not exist, age $\geq 18$, ECOG performance status $\_$, life expectancy of 12 weeks or longer. Eligibility criteria also included adequate bone marrow, hepatic, and renal function [leukocytes $\geq 3,000 / \mu 1$, absolute neutrophil count (ANC) $\geq 1.500 / \mu 1$, platelet count $\geq 100,000 / \mu 1$, glucose lower than $200 \mathrm{mg} /$ $\mathrm{dl}$, normal bilirubin level, AST or ALT levels $<2.5 \times$ the upper limit of normal (ULN), creatinine within normal limits or creatinine clearance $\succeq 60 \mathrm{ml} / \mathrm{min} / 1.73 \mathrm{~m}^{2}$ for patients with creatinine levels above institutional normal limits], and amylase levels $<2.0 \times$ the ULN. FEV $1>75 \%$ of predicted, and adequate respiratory function tests (including a DLCO > $75 \%$ of predicted) were also required. Patients could have received any prior chemotherapy or radiation therapy, as long as it was terminated 28 days prior to study initiation. Patients who had not recovered from adverse events due to agents administered more than 4 weeks earlier and those with clinically significant and/or uncontrolled medical conditions were excluded. A special focus was put on patients with diabetes mellitus, symptomatic coronary artery disease, or pulmonary dysfunction. The institutional review board of our institution granted protocol approval. Patients were required to provide written informed consent prior to enrollment into the study.

\section{Study objectives}

The primary objectives of the trial were to determine the MTD and recommended phase II doses of UCN-01 in combination with irinotecan, and to characterize the PKs of UCN-01 and irinotecan when given in combination. The secondary objectives of the trial included gaining preliminary evidence for anti-tumor effect of the combination of UCN-01 and irinotecan and determining the relationship between alpha-acid glycoprotein (AAG) concentration and UCN-01 disposition. Adverse events were classified and graded according to the National Cancer Institute Common Terminology Criteria for Adverse Events (NCI 
CTCAE) version 3.0. Standard RECIST were used to define response or progression [31]. Patients were considered evaluable for toxicity and efficacy once therapy was initiated.

\section{Treatment plan}

7-Hydroxystaurosporine was supplied by the National Cancer Institute, Division of Cancer Treatment and Diagnosis, Cancer Therapy Evaluation Program. Because of the potential for venous irritation from $\mathrm{UCN}-01$, a central port was inserted before initiation of therapy. Treatment consisted of UCN-01 administered IV on day 1 and irinotecan administered IV on days 1 and 8, both in an outpatient setting. Starting doses of UCN-01 and irinotecan were 50 and $60 \mathrm{mg} / \mathrm{m}^{2}$, respectively. Due to its prolonged half-life, the UCN-01 dose was reduced by $50 \%$ for cycles 2 and beyond. On day 1, irinotecan was administered over 90 min followed by UCN-01 as a 3-h infusion. A cycle consisted of 21 days. Prophylactic antiemetics included IV 5- $\mathrm{HT}_{3}$ antagonists and a single dose of glucocorticoids (dexamethasone $10 \mathrm{mg}$ IV) prior to chemotherapy. In the absence of treatment delays due to adverse events, treatment was administered until disease progression, intercurrent illness that prevented further administration of treatment, unacceptable adverse event(s), or withdrawal of consent. Re-treatment on day 22 required an ANC $\geq 1.500 / \mu 1$, platelet count $\geq 100,000 / \mu 1$, a creatinine clearance rate $>50 \mathrm{ml} / \mathrm{min}$, and resolution of all non-hematological toxicities (except alopecia and fatigue) to baseline or less than grade 1. In case of a delay longer than 14 days, the patient was removed from the study. The dose escalation scheme is shown in Table 1.

\section{Follow-up and monitoring}

Toxic events were recorded on a continuous basis, and followed until they were resolved to baseline or less than grade 1. Immediately prior to study entry, patients had a complete clinical history and physical examination, vital sign assessment, complete blood counts, serum biochemistry tests (albumin, alkaline phosphatase, total bilirubin, bicarbonate, BUN, calcium, chloride, creatinine, glucose, LDH, phosphorus, potassium, total protein, AST, ALT, sodium, magnesium, amylase), electrocardiogram, chest X-ray, pulmonary function test, and a disease assessment by computed tomography (CT) scan. CT scan of disease sites was repeated every two cycles. Patients on this study had serum glucose measured 2 and 24 $\mathrm{h}$ after the infusion of UCN-01. History, adverse events, complete blood counts, and serum biochemistry tests were performed on a weekly basis, or when clinically indicated.

\section{Definition of dose-limiting toxicity and maximum tolerated dose}

Non-hematologic DLT was defined as any grade 3 toxicity with the exception of diarrhea or nausea/vomiting, or any grade 4 toxicity during the first cycle. Grade 3 diarrhea lasting more than $24 \mathrm{~h}$ despite antidiarrheals and grade 3 nausea/vomiting despite optimal antiemetics were also considered a DLT. Hematologic DLT was defined as any grade 4 hematologic toxicity except neutropenia also during the first cycle. Patients with grade 4 neutropenia for $>5$ days or presenting with neutropenic fever were classified as a DLT. Patients with thrombocytopenia $<25,000 / \mu 1$ regardless of duration was classified as a DLT. An accelerated titration design was used for dose escalation, where one patient was treated at each dose level until occurrence of grade 2 or higher toxicity; when grade 2 toxicity occurred the cohort was expanded to a total of three patients. After this three patients were treated at each dose level. If no DLT was encountered in the three patients in cycle 1 , dose escalation was allowed. If one of the three patients experienced a DLT, three more patients were to be enrolled at the same dose level and if none of these three additional patients experienced a DLT, dose escalation was allowed. The MTD was defined as the highest dose tested in which fewer than 33\% of patients experienced DLT attributable to the study drug(s), when at least six patients were treated at that dose and were evaluable for toxicity. The MTD is the recommended Phase II dose for the combination of UCN-01 and irinotecan at the tested schedule. 


\section{Pharmacokinetic sampling and analytic assay for irinotecan}

Pharmacokinetic studies were done in all patients during cycle 1 day 1 and cycle 1 day 8 . Blood samples were collected at the following times: pretreatment; at $0.5 \mathrm{~h}$ during the irinotecan infusion; $5 \mathrm{~min}$ before the end of infusion; and postinfusion at $0.17,0.5,1,2,4$, $7.5,24,48$, and $72 \mathrm{~h}$ after the end of the infusion. Blood samples were collected in heparinized tubes and centrifuged at $1,000 \times g$ for $10 \mathrm{~min}$, and plasma was frozen at $-70^{\circ} \mathrm{C}$ until the time of analysis. Total plasma concentrations (i.e., the total of lactone and carboxylate) of irinotecan and its metabolites SN-38, SN-38 glucuronide (SN-38G), and APC were determined by reverse-phase high-performance liquid chromatography with fluorescence detection using a modification of a procedure described previously $[8,35]$. Irinotecan, SN-38, SN-38G, and APC were quantitated over the range of 10-3,000, 2-600, $100-600$, and $2-600 \mathrm{ng} / \mathrm{ml}$, respectively. SN-38G was measured indirectly by quantitation of the peak area at the retention time of SN-38G using the SN-38 calibration curve as previously described $[8,35]$. A limited amount of $\mathrm{SN}-38 \mathrm{G}$ was available to confirm the retention time of this metabolite (Aventis Pharma, Vitro-sur-Seine Cedex, France). Quality assurance samples were assayed with each analytic run and were within $15 \%$ of the nominal concentration.

\section{Pharmacokinetic sampling and analytic assay for UCN-01}

Pharmacokinetic studies were done in all patients during cycle 1. Blood samples were collected at the following times: pretreatment; at 0.5 , and $1.5 \mathrm{~h}$ during the infusion of $\mathrm{UCN}-01 ; 5 \mathrm{~min}$ before the end of infusion; and postinfusion at $0.5,2,4,24,48$, and $72 \mathrm{~h}$ after the end of the infusion. Samples were also obtained at 1 and 2 weeks after the end of the infusion and immediately before the second infusion. Blood samples were collected in heparinized tubes and centrifuged at $1,000 \times g$ for $10 \mathrm{~min}$, and plasma was frozen at $-70^{\circ} \mathrm{C}$ until the time of analysis. Plasma samples were analyzed using a modification of a highperformance liquid chromatography method using an UV spectrophotometer [9]. UCN-01 plasma concentrations were quantitated over the range of $0.1-100 \mu \mathrm{mol} / \mathrm{l}$. Quality assurance samples were assayed with each analytic run and were within $15 \%$ of the nominal concentration.

\section{Pharmacokinetic data analysis}

Individual plasma concentrations of irinotecan and metabolites were analyzed using noncompartmental methods as implemented in the computer software program WinNonlin version 5.0 (Pharsight, Inc., Mountain View, CA, USA). The maximum plasma concentration $\left(C_{\max }\right)$ was the observed value. The area under the plasma concentration-time curve (AUC) value was calculated to the last quantifiable sample $\left(\mathrm{AUC}_{\text {last }}\right)$ by use of the linear/log trapezoidal rule. The AUC values were extrapolated to infinity $\left(\mathrm{AUC}_{\mathrm{inf}}\right)$ by dividing the last quantifiable concentration by the terminal disposition rate constant $\left(\lambda_{\mathrm{z}}\right)$, which was determined from the slope of the terminal phase of the concentration-time profile with a $1 /$ [concentration] $]^{2}$ weight. The terminal half-life $\left(T_{1 / 2}\right)$ was calculated as 0.693 divided by $\lambda_{\mathrm{z}}$. The clearance $(\mathrm{Cl})$ was calculated by dividing the dose administered by $A U C_{\text {inf. }}$ Metabolic ratios were calculated from plasma AUC values and included the relative extent of conversion of irinotecan to its active metabolite $\mathrm{SN}-38$ (i.e., $\mathrm{AUC}_{\mathrm{SN}-38} /$ $\mathrm{AUC}_{\text {irinotecan }}$ ), the relative extent of glucuronidation of SN-38 (i.e., $\mathrm{AUC}_{\mathrm{SN}-38 \mathrm{G}} / \mathrm{AUC}_{\mathrm{SN}-38}$ ), and the relative extent of metabolism of irinotecan to $\mathrm{APC}$ (i.e., $\mathrm{AUC}_{\mathrm{APC}} / \mathrm{AUC}_{\text {irinotecan }}$ ).

7-Hydroxystaurosporine PKs were determined using a two-compartmental linear model. Individual PK parameters were estimated by the method of weighed least-squares regression as implemented in ADAPT II release 5.0 [6]. The model-fit parameters included $V_{\mathrm{c}}, K_{12}$, $K_{21}$, and $K_{\mathrm{e}}$. Calculated secondary PK variables included $V_{\mathrm{ss}}, t_{1 / 2, a}, t_{1 / 2, \beta}$, and $\mathrm{Cl}_{\mathrm{s}}$. AUC was 
calculated as dose divided by $\mathrm{Cl}_{\mathrm{s}} . C_{\max }$ values were obtained from the concentration-time data at the end of the drug infusion.

\section{Statistical analysis}

Univariate linear correlation analysis was used to assess the relationship between body surface area (BSA) and UCN-01 AUC. ANOVA was used to determine the association among patient demographics and UCN-01 exposure and worst grade of hypotension and hyperglycemia during course 1 . The method of Tukey-Kramer was used to adjust for multiple comparisons of mean values. The association between categorical variables was assessed using Fisher's exact test. Statistical analysis was done using JMP Statistical Discovery Software version 3.2.6 (SAS Institute, Cary, NC, USA). The a priori level of significance was $P<0.05$.

\section{Results}

\section{Patient characteristics}

Between January 2003 and January 2006, 16 patients with advanced solid tumors were accrued into the study. Strict entry criteria significantly hindered accrual. All patients were assessable for toxicity, and response could be evaluated in 14 patients. Demographic and clinical characteristics of the subjects are summarized in Table 2. A total of 76 cycles of the study regimen were delivered (median, two cycles; range 1-16).

\section{Dose escalation process}

Starting doses of UCN01 and irinotecan were 50 and $60 \mathrm{mg} / \mathrm{m}^{2}$, respectively (Table 1). No grade 2 or greater toxicity was observed in the first patient at dose level 1 , thus dose level 2 was opened, where UCN-01 was increased to $70 \mathrm{mg} / \mathrm{m}^{2}$ (Table 1). No DLTs were observed in dose level 2 but the cohort was increased to 3 due to a grade 2 toxicity, and thus accrual continued in dose level 3, where UCN-01 dose was escalated to $90 \mathrm{mg} / \mathrm{m}^{2}$. The third patient at dose level 3 experienced a grade 3 hypophosphatemia during cycle 1. Expansion was initiated but the fourth patient at dose level 3 experienced an episode of grade 3 hypophosphatemia, and thus this dose level was not considered safe and no further patients were treated. Both patients were reduced to the prior dose level and continued therapy for an additional cycle without recurrence of the event. Three more patients were entered at dose level 2, without any DLT. In an attempt to deliver an irinotecan dose closer to the singleagent dose, the protocol was amended and accrual continued in dose level 4, where UCN-01 was kept at $70 \mathrm{mg} / \mathrm{m}^{2}$ and irinotecan dose was escalated to $90 \mathrm{mg} / \mathrm{m}^{2}$. The first patient at dose level 4 experienced grade 3 hypophosphatemia and grade 4 hyperglycemia and expansion to six patients was planned. However, the fourth patient treated at this dose level had a grade 3 hypophosphatemia and the fifth subject was hospitalized with grade 4 febrile neutropenia. Dose level 2 (already expanded to six patients and with 0/6 DLTs) consisting of $70 \mathrm{mg} / \mathrm{m}^{2}$ of UCN-01 on day 1 and $60 \mathrm{mg} / \mathrm{m}^{2}$ of irinotecan on days 1 and 8 of a 21-day cycle was defined as the MTD.

\section{Toxicity}

Treatment was generally well tolerated and in concordance with the expected side effect profile of both agents. The toxic events experienced during cycle 1 or during all cycles of therapy and thought to be possibly, probably, or likely related to UCN-01 and/or irinotecan are listed in Tables 3 and 4, respectively. The most common adverse events were fatigue, diarrhea, nausea, vomiting, anorexia, hyperglycemia, hypotension, and abdominal cramps. Diarrhea and abdominal cramps are thought to be related to irinotecan, whereas nausea, 
vomiting, hyperglycemia, and hypotension (and lightheadedness) have been consistently associated with UCN-01.

A significant number of patients had laboratory abnormalities which constituted the majority of the grade 3-4 events, particularly during the first cycle. Eight patients experienced hyperglycemia in 11 courses, of which two were grade 3 and one grade 4 . Patients were successfully managed with hypoglycemic agents and did not require dose reductions. Five patients had significant hypophosphatemia in 13 courses, of which five were grade 3; four of these grade 3 events occurred during the first cycle, constituting the principal dose-limiting toxicity of the combination, as detailed above. After dose reductions in the affected patients, the events did not recur. Infusion-related nausea, vomiting, headache, and lightheadedness were also likely UCN-01-related toxicities that were frequently observed. Interestingly, whereas nausea/vomiting occurred repeatedly over subsequent cycles of therapy (successfully managed with increased antiemetic therapy), headache and light-headedness were almost exclusively documented during the first cycle. Despite the occurrence of hyperglycemia, the reason for maintaining a single dose of dexamethasone prior to UCN-01 in subsequent cohorts were that nausea and vomiting represented a toxicity that was more relevant clinically, and a single corticosteroid dose in non-diabetic subjects was not felt to substantially increase the risk of a severe hyperglycemic event. Other less frequent toxicities observed were hypokalemia, hyponatremia, stomatitis, fever, and facial flushing. Few neurologic and no pulmonary events were recorded. One patient presented with a transient, grade 2 episode of confusion that was attributed to concomitant medication and one patient presented grade 1 numbness in the perioral area that subsided spontaneously. A small proportion of patients experienced hematologic toxicity, although one case of grade 4 febrile neutropenia was documented. This event had an uncomplicated course and the patient recovered shortly thereafter, but was taken off the study for disease progression and thus the impact of reducing the dose could not be evaluated.

Patient demographics (age, BSA, and sex), AAG concentrations, and UCN-01 plasma exposure $\left(C_{\max }\right.$ and AUC) were not related to worst grade of hypotension or hyperglycemia during course $1(P>0.08)$. UCN-01 AUC was related to worst grade of hypotension during course $1(P=0.02)$.

\section{Activity}

Of the 16 patients, 14 are assessable for response. In one subject progression was suspected clinically and documented radiologically after one course of treatment, and seven had progressive disease upon reevaluation after two cycles. The other six patients had stable disease as best response, and there were four patients (two ECOG 0, two ECOG 1) that received ten or more cycles of the study regimen (base of tongue carcinoma and biliary adenocarcinoma ten cycles each, medullary thyroid cancer 11 cycles, and colorectal adenocarcinoma 16 cycles), without requiring dose reductions for acute or chronic toxicities.

\section{Pharmacokinetics}

Plasma samples were obtained from all 16 patients for UCN-01, and PK data were evaluable for all patients for UCN-01 and 15 patients for irinotecan. UCN-01 plasma PK variables are listed in Table 5. At the MTD, $70 \mathrm{mg} / \mathrm{m}^{2}$ administered over $3 \mathrm{~h}$, average ( \pm standard deviation) values for $V_{\mathrm{ss}}, t_{1 / 2, \beta}$, and $\mathrm{Cl}_{\mathrm{s}}$ were $14.3 \pm 5.91,292.0 \pm 135.7 \mathrm{~h}$, and $0.045 \pm$ $0.038 \mathrm{l} / \mathrm{h}$, respectively. At this dose level, average ( \pm standard deviation) values for $C_{\max }$ and AUC were $34.7 \pm 16.9 \mu \mathrm{mol} / \mathrm{l}$ and $10,061 \pm 5,106 \mu \mathrm{mol} \mathrm{h} / 1$, and values for these exposure variables varied 4.8 - and 14.5 -fold, respectively. 
Because the dose of UCN-01 is calculated based on a patient's BSA, the relationship between BSA and UCN-01 PK variables were assessed. BSA was associated with neither UCN-01 $V_{\mathrm{SS}}(R=0.05 ; P=0.85)$ nor $\mathrm{Cl}_{\mathrm{S}}(R=0.37 ; P=0.16)$. To search for other clinical variables that may be related to UCN-01 exposure, the relationships between pre-treatment AAG and UCN-01 PK variables were also assessed. Pre-treatment AAG concentrations were correlated with both UCN-01 $V_{\mathrm{ss}}(R=0.59 ; P=0.06)$ and $\mathrm{Cl}_{\mathrm{S}}(R=0.56 ; P=0.07)$ but did not reach significance in this small population. Co-administration of irinotecan does not appear to alter UCN-01 PKs.

Irinotecan plasma PK variables are listed in Table 6. When using a paired $t$-test to compare AUC and $C_{\max }$ on both day 1 and day 8, there was a significant reduction in SN-38 AUC ( $P$ $=0.03)$, APC AUC $(P=0.04)$, and APC $C_{\max }(P=0.03)$. This translated to a $50 \%$ decrease in SN38:irinotecan AUC ratio $(P=0.03)$. A similar trend was observed with the APC:irinotecan AUC ratio $(P=0.06)$ but it did not reach statistical significance. The alteration was not observed in a significant chance in irinotecan or metabolite half-lives.

\section{Discussion}

7-Hydroxystaurosporine is a selective inhibitor of a number of serine-threonine kinases, particularly PKC, Chk1, and PDK1. Despite its level of preclinical activity against multiple in vivo models, the two reported phase I trials encompassing a total of 71 patients (17 treated at the respective MTDs) showed modest antitumor activity, with one melanoma patient showing a partial response $[9,25]$. Thus, rational combinations with cytotoxic agents have been conducted. Based on the observed preclinical synergy and the potentially nonoverlapping toxicity profiles, $\mathrm{UCN}-01$ and irinotecan were combined in this dose-finding phase I study designed to determine the MTD, acute, and chronic toxicity profile, and PK of $\mathrm{UCN}-01$ and irinotecan. The escalation design successfully increased UCN-01 first, but neither further increases in UCN-01, nor escalations of irinotecan, were well tolerated. Thus dose level 2, consisting of $70 \mathrm{mg} / \mathrm{m}^{2}$ of UCN-01 on day 1 and $60 \mathrm{mg} / \mathrm{m}^{2}$ of irinotecan on days 1 and 8 of a 21-day cycle, was defined as the MTD. At this dose level no DLTs occurred in six treated patients. In this trial the accelerated titration design did not seem advantageous, possibly due to the relative (onefold) proximity of the combination starting doses to the single-agent tolerated doses.

Metabolic toxicities such as hypophosphatemia and hyperglycemia constituted most of the grade 3-4 events, and were defined as dose-limiting. Hypophosphatemia had not previously been identified as dose limiting and may be unique to this combination. Hyperglycemia has previously been described as due to insulin resistance and may be related to UCN-01 inhibiting PDK signaling [20]. As expected with irinotecan, diarrhea, and abdominal disturbances were prevalent but easily managed, and no potentiation by UCN-01 was seen. Interestingly, few neurological and pulmonary events occurred, suggesting a close dosedependency of these toxicities with UCN-01. Hematologic toxicity was notably mild, with the exception of one episode of grade 4 febrile neutropenia. Another remarkable aspect was that none of the four patients who received ten or more cycles of the study regimen required dose reductions for acute or chronic toxicities, highlighting the tolerability of the combination at the evaluated doses.

The molecular basis of these UCN-01-related metabolic toxicities is currently undetermined. Parathyroid hormone is the main phosphate regulation mechanism, and inhibits proximal tubular reabsorption of phosphate by retrieval of type IIa Na- $\mathrm{PO}_{4}$ cotransporters (Npt2a) from the renal tubule membrane. This process is mediated by a complex equilibrium between the activation of both protein kinases A and C [32] that ultimately converge on the extracellular signal-regulated kinase/mitogen-activated protein kinase (ERK/MAPK) 
pathway [2]. Therefore, UCN-01-mediated PKC inhibition/modulation may disrupt this equilibrium and induce the observed alterations in phosphate and calcium levels. $\mathrm{PKC}$ is also involved at several steps in glucose metabolism. PKC regulates insulin action and degradation by interacting with insulin receptor substrates [24]. Glucose absorption control is mediated in part by PKC and ERK/MAPK-dependent activation and recruitment of glucose transporters to the intestinal membrane $[13,18]$. Glucose and fatty acid uptake by tissues is also mediated in part by PKC modulation [21], and PKC has been proposed to have a pathogenic impact in diabetes, as PKC-beta and other PKC isoenzymes have been implicated in the loss of endothelial barrier function in diabetic micro-angiopathy [15]. The complexities of these control mechanisms are evidenced by the observation of opposite effects on the same pathway, as although hyperglycemia was predominant, hypoglycemia was also documented.

Similar to results from the phase I study of 72-h infusions [25] and shorter infusion times of 1-3 h [9], UCN-01 exhibited a long half-life (292 h), low clearance (0.045 l/h), and marked variability in AUC values ( 15 -fold). The long half-life has been attributed in part to highaffinity binding to AAG which only has a half-life of (3-5 days) [10, 25]. As with other studies, we saw no relationship between AAG and several PK parameters [9, 28]. Not unexpectedly, there were no correlations between increasing UCN-01 exposure and increasing grade of toxicity which is consistent with previous single agent trials [9].

Since irinotecan is highly susceptible to CYP3A4 and ABCB1 inhibition [17] and induction [22] in humans, alterations to the PKs are anticipated with agents that modulate these proteins. A limitation of the current study was not performing a PK assessment of irinotecan alone to determine the true interaction since $\mathrm{UCN}-01$ was present during both day 1 and day 8. Despite this, a significant decrease in both CYP3A4 and carboxylesterase-mediated pathways was observed by 8 days of continual exposure to UCN-01. A previous study demonstrated that $\mathrm{ABCB} 1$ was not induced in the presence of $\mathrm{UCN}-01$ in the lung cancer cell line A549 [5]. Currently, there appears to be no published literature on the alteration of CYP3A4 with UCN-01. Therefore, the mechanisms behind this possible drug interaction are unknown.

A study combining of UCN-01 and topotecan has been reported, showing good tolerance and preliminary evidence of clinical activity in platinum-refractory ovarian cancer patients [14]. The recommended phase II schedule was UCN-01 $70 \mathrm{mg} / \mathrm{m}^{2}$ as a 3-h infusion on day 1 , followed by topotecan $1.0 \mathrm{mg} / \mathrm{m}^{2}$ infused over 30 minutes on days $1-5$. The authors reported a profile of non-hematological toxicities that resembles the currently presented data, consisting of hyperglycemia, infection, coagulation abnormalities, fatigue, hypotension, nausea, and hypomagnesemia. However, hematologic toxicities occurred universally in that study, supporting the known higher myelotoxicity of topotecan when compared with irinotecan. A subsequent phase II study of this combination in patients with ovarian cancer is underway. Unfortunately the PK analysis did not include topotecan, and thus we cannot ascertain whether the interaction observed with irinotecan is agent-or familyspecific.

The significance and potential use of this combination is uncertain, as it is not clear at this point whether UCN-01 will continue its clinical development. However, as a class of agents PKC inhibitors are showing promise. Enzastaurin is an orally available PKC beta inhibitor that displays proapoptotic properties against a spectrum of malignancies and is currently being investigated in clinical trials $[4,12]$.

In summary, the combination of UCN-01 and irinotecan at clinically relevant doses for both agents was shown to have an acceptable toxicity profile in patients with advanced solid 
malignancies. While UCN-01 was not affected by irinotecan, irinotecan and SN38 exposure decreased when administered in combination with UCN-01.

\section{Acknowledgments}

UO1 program CA 70095, Commonwealth Foundation for Cancer Research, American Society of Clinical Oncology Young Investigator Award (A.J.).

\section{References}

1. Akinaga S, Nomura K, Gomi K, Okabe M. Effect of UCN-01, a selective inhibitor of protein kinase $\mathrm{C}$, on the cell-cycle distribution of human epidermoid carcinoma, A431 cells. Cancer Chemother Pharmacol. 1994; 33:273-280. [PubMed: 7506638]

2. Bacic D, Schulz N, Biber J, Kaissling B, Murer H, Wagner CA. Involvement of the MAPK-kinase pathway in the PTH-mediated regulation of the proximal tubule type IIa Na+/Pi co-transporter in mouse kidney. Pflugers Arch. 2003; 446:52-60. [PubMed: 12690463]

3. Bredel M, Pollack IF, Freund JM, Rusnak J, Lazo JS. Protein kinase C inhibition by UCN-01 induces apoptosis in human glioma cells in a time-dependent fashion. J Neurooncol. 1999; 41:9-20. [PubMed: 10222418]

4. Carducci MA, Musib L, Kies MS, et al. Phase I dose escalation and pharmacokinetic study of enzastaurin, an oral protein kinase $\mathrm{C}$ beta inhibitor, in patients with advanced cancer. J Clin Oncol. 2006; 24:4092-4099. [PubMed: 16943527]

5. Courage C, Bradder SM, Jones T, Schultze-Mosgau MH, Gescher A. Characterisation of novel human lung carcinoma cell lines selected for resistance to anti-neoplastic analogues of staurosporine. Int J Cancer. 1997; 73:763-768. [PubMed: 9398059]

6. D'Argenio DZ, Schumitzky A. A program package for simulation and parameter estimation in pharmacokinetic systems. Comput Programs Biomed. 1979; 9:115-134. [PubMed: 761456]

7. Dasmahapatra GP, Didolkar P, Alley MC, Ghosh S, Sausville EA, Roy KK. In vitro combination treatment with perifosine and $\mathrm{UCN}-01$ demonstrates synergism against prostate (PC-3) and lung (A549) epithelial adenocarcinoma cell lines. Clin Cancer Res. 2004; 10:5242-5252. [PubMed: 15297428]

8. de Bruijn P, Willems EW, Loos WJ, Verweij J, Sparreboom A. determination of the irinotecan metabolite 7-ethyl-10-O-glucuronyl-camptothecin in human samples. Anal Biochem. 2004; 328:8486. [PubMed: 15081912]

9. Dees EC, Baker SD, O'Reilly S, et al. A phase I and pharmacokinetic study of short infusions of UCN-01 in patients with refractory solid tumors. Clin Cancer Res. 2005; 11:664-671. [PubMed: 15701854]

10. Fuse E, Tanii H, Kurata N, et al. Unpredicted clinical pharmacology of UCN-01 caused by specific binding to human alpha1-acid glycoprotein. Cancer Res. 1998; 58:3248-3253. [PubMed: 9699650]

11. Graves PR, Yu L, Schwarz JK, et al. The Chk1 protein kinase and the Cdc $25 \mathrm{C}$ regulatory pathways are targets of the anti-cancer agent UCN-01. J Biol Chem. 2000; 275:5600-5605. [PubMed: 10681541]

12. Green LJ, Marder P, Ray C, et al. Development and validation of a drug activity biomarker that shows target inhibition in cancer patients receiving enzastaurin, a novel protein kinase C-beta inhibitor. Clin Cancer Res. 2006; 12:3408-3415. [PubMed: 16740765]

13. Helliwell PA, Rumsby MG, Kellett GL. Intestinal sugar absorption is regulated by phosphorylation and turnover of protein kinase $\mathrm{C}$ betaII mediated by phosphatidylinositol 3-kinase- and mammalian target of rapamycin-dependent pathways. J Biol Chem. 2003; 278:28644-28650. [PubMed: 12766174]

14. Hotte SJ, Oza A, Winquist EW, et al. Phase I trial of UCN-01 in combination with topotecan in patients with advanced solid cancers: a Princess Margaret Hospital Phase II Consortium study. Ann Oncol. 2006; 17:334-340. [PubMed: 16284058] 
15. Idris I, Gray S, Donnelly R. Protein kinase C-beta inhibition and diabetic microangiopathy: effects on endothelial permeability responses in vitro. Eur J Pharmacol. 2004; 485:141-144. [PubMed: 14757134]

16. Kawakami K, Futami H, Takahara J, Yamaguchi K. UCN-01, 7-hydroxyl-staurosporine, inhibits kinase activity of cyclin-dependent kinases and reduces the phosphorylation of the retinoblastoma susceptibility gene product in A549 human lung cancer cell line. Biochem Biophys Res Commun. 1996; 219:778-783. [PubMed: 8645257]

17. Kehrer DF, Mathijssen RH, Verweij J, de Bruijn P, Sparreboom A. Modulation of irinotecan metabolism by ketoconazole. J Clin Oncol. 2002; 20:3122-3129. [PubMed: 12118026]

18. Kellett GL. The facilitated component of intestinal glucose absorption. J Physiol. 2001; 531:585595. [PubMed: 11251042]

19. Komander D, Kular GS, Bain J, Elliott M, Alessi DR, Van Aalten DM. Structural basis for UCN-01 (7-hydroxystaurosporine) specificity and PDK1 (3-phosphoinositide-dependent protein kinase-1) inhibition. Biochem J. 2003; 375:255-262. [PubMed: 12892559]

20. Kortmansky J, Sauter N, O'Reilly E. Management of hyperglycemia in patients with metastatic pancreatic cancer receiving UCN-01 and fluorouracil (2004 ASCO annual meeting proceedings (post-meeting edn)). J Clin Oncol. 2004; 22(14S (July 15 Suppl)):2140.

21. Luiken JJ, Coort SL, Koonen DP, Bonen A, Glatz JF. Signalling components involved in contraction-inducible substrate uptake into cardiac myocytes. Proc Nutr Soc. 2004; 63:251-258. [PubMed: 15294039]

22. Mathijssen RH, Verweij J, de Bruijn P, Loos WJ, Sparreboom A. Effects of St John's wort on irinotecan metabolism. J Natl Cancer Inst. 2002; 94:1247-1249. [PubMed: 12189228]

23. Monks A, Harris ED, Vaigro-Wolff A, Hose CD, Connelly JW, Sausville EA. UCN-01 enhances the in vitro toxicity of clinical agents in human tumor cell lines. Invest New Drugs. 2000; 18:95107. [PubMed: 10857990]

24. Oriente F, Andreozzi F, Romano C, et al. Protein kinase C-alpha regulates insulin action and degradation by interacting with insulin receptor substrate-1 and 14-3-3 epsilon. J Biol Chem. 2005; 280:40642-40649. [PubMed: 16216880]

25. Sausville EA, Lush RD, Headlee D, et al. Clinical pharmacology of UCN-01: initial observations and comparison to preclinical models. Cancer Chemother Pharmacol. 1998; 42(Suppl):S54-S59. [PubMed: 9750030]

26. Sausville EA, Arbuck SG, Messmann R, et al. Phase I trial of 72-hour continuous infusion UCN-01 in patients with refractory neoplasms. J Clin Oncol. 2001; 19:2319-2333. [PubMed: 11304786]

27. Seynaeve CM, Stetler-Stevenson M, Sebers S, Kaur G, Sausville EA, Worland PJ. Cell cycle arrest and growth inhibition by the protein kinase antagonist UCN-01 in human breast carcinoma cells. Cancer Res. 1993; 53:2081-2086. [PubMed: 7683251]

28. Sparreboom A, Chen H, Acharya MR, et al. Effects of alpha1-acid glycoprotein on the clinical pharmacokinetics of 7-hydroxystaurosporine. Clin Cancer Res. 2004; 10:6840-6846. [PubMed: 15501960]

29. Takahashi I, Kobayashi E, Asano K, Yoshida M, Nakano H. UCN-01, a selective inhibitor of protein kinase C from Streptomyces. J Antibiot (Tokyo). 1987; 40:1782-1784. [PubMed: 3429345]

30. Takahashi I, Saitoh Y, Yoshida M, et al. UCN-01 and UCN-02, new selective inhibitors of protein kinase C. II. Purification, physico-chemical properties, structural determination and biological activities. J Antibiot (Tokyo). 1989; 42:571-576. [PubMed: 2656615]

31. Therasse P, Arbuck SG, Eisenhauer EA, et al. New guidelines to evaluate the response to treatment in solid tumors. European Organization for Research and Treatment of Cancer, National Cancer Institute of the United States, National Cancer Institute of Canada. J Natl Cancer Inst. 2000; 92:205-216. [PubMed: 10655437]

32. Traebert M, Volk1 H, Biber J, Murer H, Kaissling B. Luminal and contraluminal action of 1-34 and 3-34 PTH peptides on renal type IIa Na-P(i) cotransporter. Am J Physiol Renal Physiol. 2000; 278:F792-F798. [PubMed: 10807591] 
33. Tse AN, Schwartz GK. Potentiation of cytotoxicity of topoisomerase i poison by concurrent and sequential treatment with the checkpoint inhibitor UCN-01 involves disparate mechanisms resulting in either p53-independent clonogenic suppression or p53-dependent mitotic catastrophe. Cancer Res. 2004; 64:6635-6644. [PubMed: 15374978]

34. Usuda J, Saijo N, Fukuoka K, et al. Molecular determinants of UCN-01-induced growth inhibition in human lung cancer cells. Int J Cancer. 2000; 85:275-280. [PubMed: 10629089]

35. van Erp NP, Baker SD, Zhao M, et al. Effect of milk thistle (Silybum marianum) on the pharmacokinetics of irinotecan. Clin Cancer Res. 2005; 11:7800-7806. [PubMed: 16278402]

36. Yu Q, La Rose J, Zhang H, Takemura H, Kohn KW, Pommier Y. UCN-01 inhibits p53 upregulation and abrogates gamma-radiation-induced $\mathrm{G}(2)-\mathrm{M}$ checkpoint independently of p53 by targeting both of the checkpoint kinases, Chk2 and Chk1. Cancer Res. 2002; 62:5743-5748.

[PubMed: 12384533] 


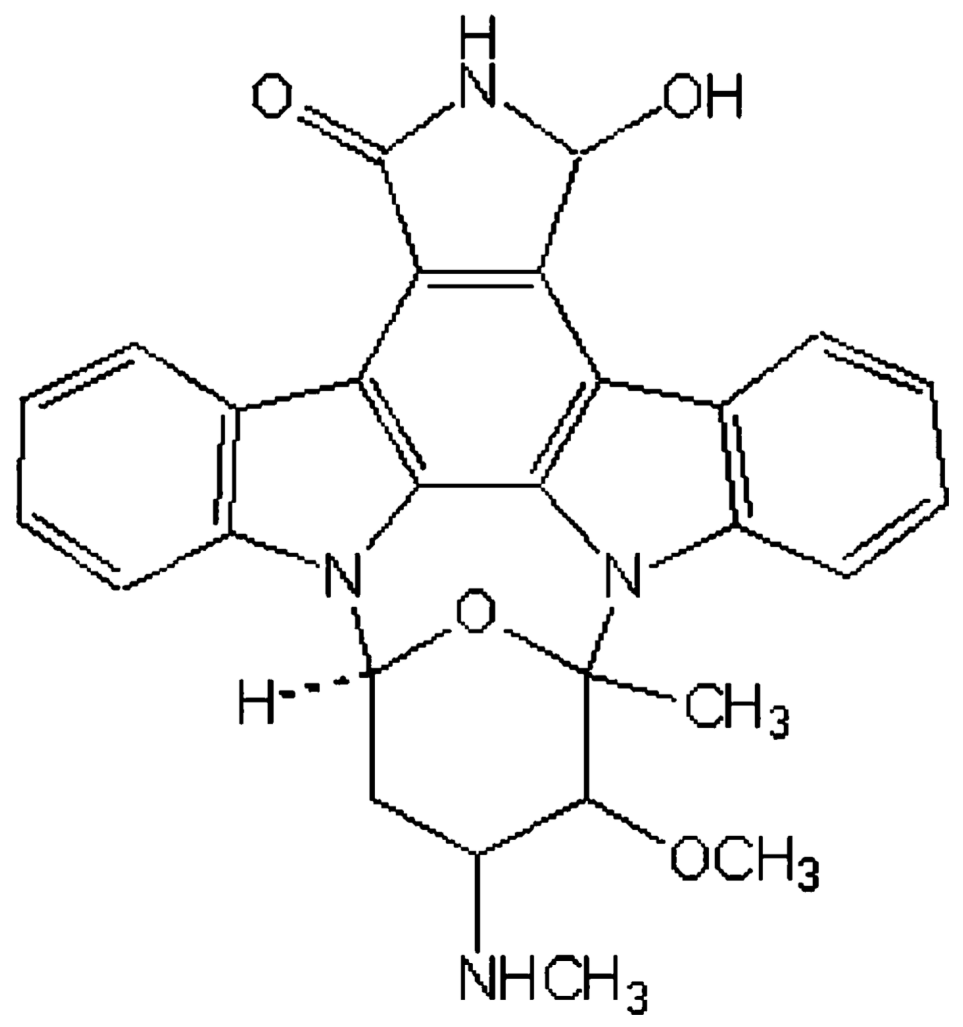

Fig. 1.

Chemical structure of UCN-01 


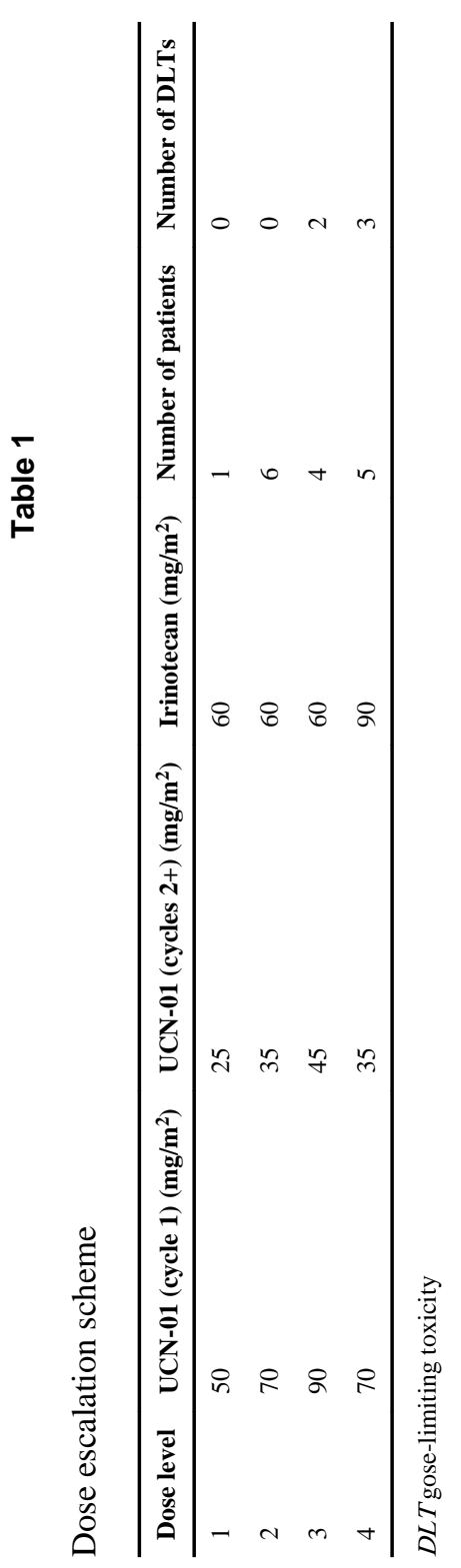

Cancer Chemother Pharmacol. Author manuscript; available in PMC 2013 January 28. 
Table 2

Patient characteristics

\begin{tabular}{ll}
\hline Characteristics & No. of patients \\
\hline No. of patients & 16 \\
Sex (male/female) & $11 / 5$ \\
Age (years), median (range) & $59(34-75)$ \\
Eastern Cooperative Oncology Group Performance Status \\
0 & 6 \\
1 & 10 \\
Primary tumor site & \\
Colorectal & 4 \\
Pancreas (adenocarcinoma) & 2 \\
Pancreas (neuroendocrine) & 1 \\
Biliary & 1 \\
Duodenal & 1 \\
Hepatocellular & 1 \\
Base of tongue (adenocystic carcinoma) & 1 \\
Mesothelioma & 1 \\
Thyroid (medullary) & 1 \\
Ovary & 1 \\
Skin (merkel cell) & 1 \\
Unknown primary (adenocarcinoma) & \\
\hline Prior regimens for advanced disease & \\
\hline & 1 \\
\hline
\end{tabular}




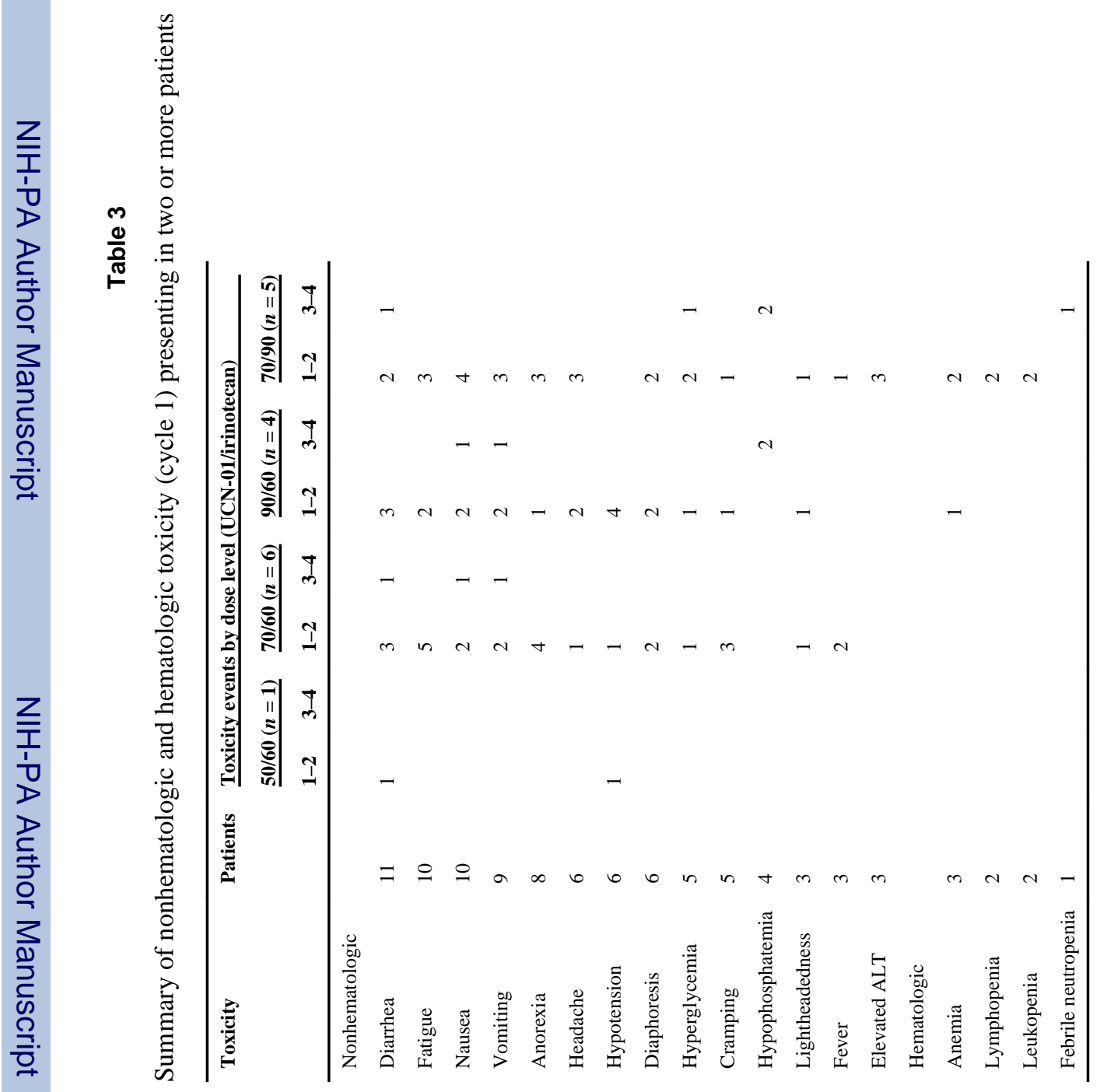




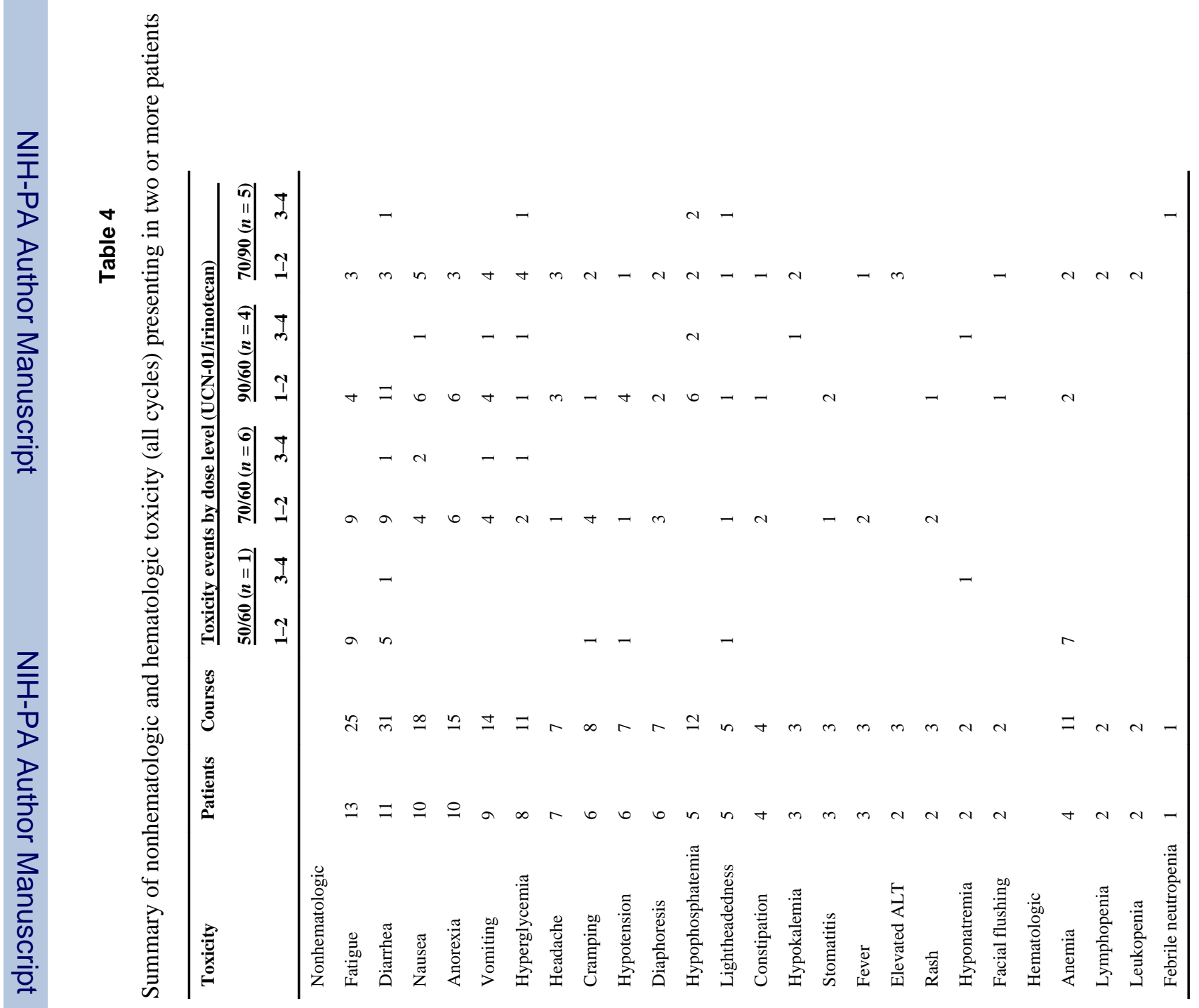

Cancer Chemother Pharmacol. Author manuscript; available in PMC 2013 January 28. 


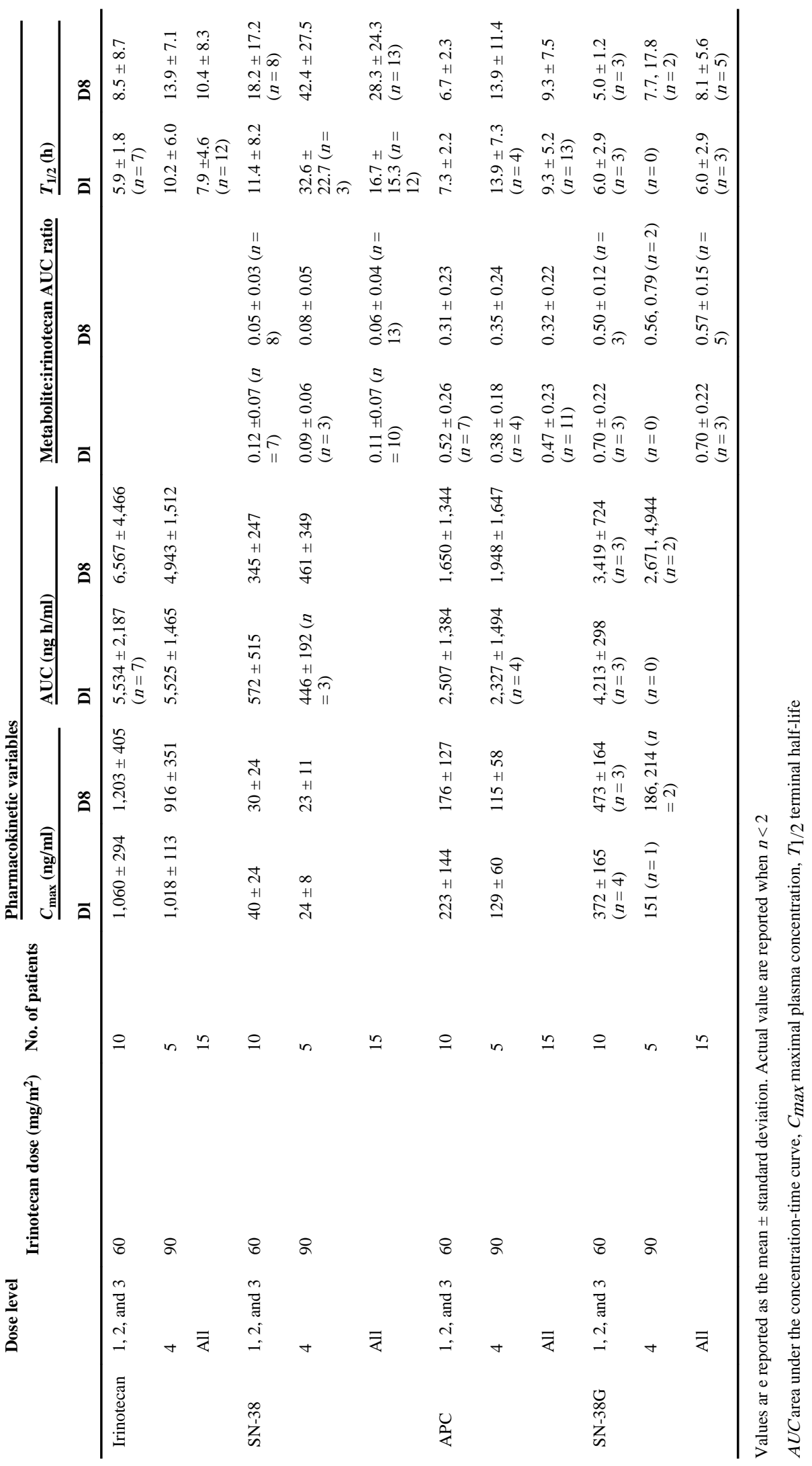

